\title{
Caracterización de las mejores prácticas educativas: UNESCO y el paradigma del aprendizaje móvil
}

\author{
L. Belén Espejo Villar \\ Ibev@usal.es \\ Universidad de Salamanca, España \\ Luján Lázaro Herrero \\ lujan@usal.es \\ Universidad de Salamanca, España \\ Gabriel Álvarez López \\ gabal@usal.es \\ Universidad de Salamanca, España

\section{Enric Prats Gil} \\ enricprats@ub.edu \\ Universidad de Barcelona, España
}

\begin{abstract}
Resumen
El trabajo presenta desde un enfoque comparado escuelas internacionales de éxito que están transformando la educación (bottom-up) a partir de su implicación con la tecnología digital. El estudio trata de mostrar un conjunto de escuelas de diferentes países (Brasil, China, España, Israel, Portugal, Reino Unido y Rusia) seleccionadas por UNESCO como iniciativas pioneras de equidad educativa por la proyección pedagógica y social que hacen del aprendizaje móvil. El artículo trata de acotar los principios causales que subyacen en las mejores prácticas escolares tal y como son conceptuadas por actores de poder (UNESCO). Se ha utilizado la metodología de análisis político del discurso combinada con el estudio de casos con el propósito de interpretar las lógicas de las que son objeto las escuelas elegidas en la transferibilidad de su éxito. Los datos reflejan el valor educativo de las tecnologías digitales en centros cuya función social se impone a la titularidad de los mismos y la soberanía de la digitalización en el gobierno de los centros educativos, al tiempo que muestran un cambio de tendencia en la caracterización de las mejores prácticas educativas, al pasar de la excelencia entendida como resultados a la excelencia delimitada como inclusión socioeducativa (procesos).
\end{abstract}

\section{Palabras Clave}

Aprendizaje móvil, tecnología digital, bien social público, éxito académico, transferencia de aprendizaje, bottom-up. 


\title{
Characterization of educational best practices: UNESCO and the mobile learning paradigm
}

\author{
L. Belén Espejo Villar \\ Ibev@usal.es \\ Universidad de Salamanca, Spain \\ Luján Lázaro Herrero \\ lujan@usal.es \\ Universidad de Salamanca, Spain \\ Gabriel Álvarez López \\ gabal@usal.es \\ Universidad de Salamanca, Spain \\ Enric Prats Gil \\ enricprats@ub.edu \\ Universidad de Barcelona, Spain
}

\begin{abstract}
The study uses a comparative approach to successful international schools that are changing education (bottom-up) using digital technology. The study aims to show a group of schools from different countries (Brazil, China, Spain, Israel, Portugal, United Kingdom and Russia), that were selected by the UNESCO as being pioneers of educational equity of mobile learning from a pedagogical and social perspective. The article discusses the potential of mobile learning in models of social inclusion, trying to narrow down the causal principles underlying school best practices as they are conceptualized by power actors (UNESCO). We used a political discourse analysis, combined with a case study for the selected schools, and we intend to describe their methods to see if their success can be transferred. The article tries to define the fundamental principles underlying in most excellent school practices as they are conceived by influential institutions (UNESCO) .The data reflect the educational capital of digital technologies in schools whose social function is imposed on their ownership and the sovereignty of digitization in the government of schools, while showing a change in trend in the characterization of educational best practices, moving from excellence understood as results to excellence delimited as socioeducational inclusion (processes).
\end{abstract}

\section{Keywords}

Mobile learning, digital technology, public social goods, academic success, learning transfer, bottom-up. 


\section{Introducción}

Asistimos a tiempos cambiantes en los que la incertidumbre que genera el dinamismo de los acontecimientos a nivel mundial obliga a respuestas rápidas de los sistemas políticos (Innerarity, 2019) y educativos. La actual declaración de pandemia por parte de la Organización Mundial de la Salud (OMS, 2020) ha forzado a las instituciones escolares a hacer uso de herramientas digitales que puedan garantizar la continuidad de la formación situando en la vanguardia educativa el aprendizaje en cualquier momento y en cualquier lugar, o lo que es lo mismo, el aprendizaje ubicuo (Burbules, 2014).

Como complemento a la escolarización presencial en contextos de difícil acceso, el aprendizaje móvil (mobile-learning) está siendo una de las apuestas más destacadas y acreditadas de la UNESCO. Así, en consonancia con la estrategia diseñada para alcanzar el ODS-4 de la Agenda 2030, que busca expandir al máximo la escolarización y elevar los niveles de aprendizaje a los sectores más vulnerables, sobre todo a aquellos que tienen más dificultad para acceder a recursos educativos, la UNESCO ha reivindicado en diversas publicaciones (2012, 2013, 2017, 2020a, 2020b) alternativas a la enseñanza presencial que además contemplen la posibilidad de formación entre los propios docentes.

Bajo estas premisas nace en el año 2016 el Proyecto UNESCO-Fazheng que se abre tanto a programas gubernamentales (top-down), como a centros educativos (bottom-up) públicos y privados, con el firme propósito de garantizar instituciones escolares inclusivas y equitativas, convirtiendo el aprendizaje móvil en un paradigma social con legitimidad para minimizar los componentes multifactoriales (culturales, socioeconómicos e institucionales) que intervienen en el proceso educativo. En este trabajo, nos centramos en el segundo tipo de experiencia (bottom-up), por la legitimidad que este enfoque narrativo, basado en la unidad escolar, proporciona en el modo de organización y funcionamiento a los centros.

En este contexto, la UNESCO abrió en el año 2018 una convocatoria relativa a la modalidad de micropolítica educativa (bottom-up), encaminada a seleccionar las mejores prácticas de aprendizaje móvil vinculadas a instituciones educativas de las que resultaron elegidas, por sus proyectos de digitalización, las prácticas de siete centros escolares (de más de 150 candidaturas presentadas). La dispersión geoterritorial de los centros seleccionados (Brasil, China, España, Israel, Portugal, Reino Unido y Rusia) incorpora a esta propuesta de digitalización dos novedades; por un lado, se convierte en un elemento de transferibilidad desde el que plantear la digitalización educativa como un componente de armonización a nivel internacional (Dale, 2005), poniendo de manifiesto la fortaleza de las redes institucionales, el aprendizaje colaborativo y equidad educativa (paradigma social), por otra parte se presenta el aprendizaje móvil como un factor legitimador de la capacidad directiva y de gestión de los centros escolares.

La dimensión macropolítica desde la que se caracteriza la digitalización en el marco de la agenda gubernamental (UNESCO) nos sumerge en el estudio de las directrices organizativas y contextuales sobre las que se están construyendo los centros educativos que han hecho del aprendizaje móvil sus señas de identidad. Presentadas por la UNESCO como las escuelas con las mejores prácticas tecnológicas, estos centros nos permiten comprender en qué se sustenta la singularidad del soporte digital (aprendizaje móvil) para retratarlo como un componente identificador de los mejores centros educativos. ¿Bajo qué premisas se construyen las que han sido conceptuadas por organismos internacionales como las mejores prácticas educativas? ¿Qué variables subyacen en la caracterización que hace la UNESCO de las mejores escuelas? ¿Podríamos pensar a partir de la selección realizada que se está imponiendo la digitalización a otros tipos de indicadores de éxito que hasta ahora han condicionado la organización y la gestión de los centros educativos?

La elección de la dimensión bottom-up en este artículo responde no sólo a la importancia que están adquiriendo los centros educativos como unidades de poder en la implementación de prácticas 
educativas digitales en un momento de suspensión de la presencialidad del aprendizaje, sino también a la necesidad de comprobar si el gobierno de los centros educativos ha dejado de construirse a partir de estándares de excelencia, para hacerlo con políticas de digitalización educativa que contribuyan a la inclusión socioeducativa. ¿Qué lecciones pueden obtenerse del estudio comparado de estas escuelas? ¿Qué caracterización se puede hacer del concepto de mejores escuelas a partir de este trabajo?

\section{Marco teórico}

\section{a. Caracterización de las mejores escuelas desde la excelencia educativa}

En los últimos años, el creciente interés surgido en torno a los centros educativos en el marco de la agenda política internacional (Darling-Hammond, et al., 2017; OCDE, 2018a, 2018b, 2016, 2012) ha presentado las instituciones escolares como una estructura de gobierno con legitimidad para la gestión y la organización curricular y con autoridad para decidir las políticas de evaluación, las actuaciones disciplinarias y de admisión, así como las relativas a las condiciones económicas del profesorado. Una línea de control con la que se ha venido identificando la supremacía instrumental que ejercen los centros educativos.

A este empoderamiento de las escuelas ha contribuido igualmente la tendencia a identificar el liderazgo educativo de los centros con sistemas educativos exitosos (Delannoy y Guzmán, 2009; Egido, 2015; Sanz y Serrano, 2017) y la propensión a pensar que la autonomía escolar se construye únicamente desde modelos de gestión basados en estándares (Ball y Yodell, 2007; Casassus, 2010; Verger y Norman, 2015) y en entornos de seguridad que son propiciados a partir de la libertad de elección (OCDE, 2018a). Todo ello ha llevado a conceptuar los centros en una dimensión formal, entendiéndolos como las nuevas unidades de gobierno (bottom-up) con autoridad para gestionar procesos en los que el foco de poder se ha ido desplazando de los niveles estatales-regionales al ámbito escolar.

Diversas investigaciones han relacionado la idea de las mejores escuelas con la excelencia académica. Los trabajos de Rostan y Vaira (2011), y los de Wößmann (2003), Wößmann, Lüdemann, Schütz, \& West (2007), constatan cómo la soberanía del centro se ha ido forjando en torno al paradigma de la cuantificación educativa, orientando sus prácticas al éxito institucional, e incrementando el desarrollo de líneas de investigación relacionadas con la búsqueda de mayores logros de aprendizaje (Kotthoff, 2011).

\section{b. Caracterización de las mejores escuelas desde la digitalización educativa}

La preocupación por la producción educativa ha habilitado recientemente otras vías de estudio (innovación, metodologías, tecnologías) con la intención de instrumentalizar la autonomía de los centros y de focalizar la transformación de las escuelas en torno a las posibilidades metodológicas y su integración en el aula (Area et al., 2020). En estos estudios relativos a metodologías y herramientas innovadoras (Araujo, Dias \& Ota, 2014; Brazuelo, Gallego y Cacheiro, 2017; Hsu, Ching, \& Snelson, 2014 y Martín-García, 2020) han comenzado a cobrar fuerza las posibilidades de la digitalización educativa, y en concreto las tesis de erigirse en un contrapoder de políticas excluyentes y selectivas. Su potencial en diferentes áreas de conocimiento, así como su correlación con el rendimiento académico y la motivación escolar (Caldeiro, Yot, y Castro, 2018; RamírezMontoya \& Ramírez-Hernández, 2016; Sánchez Prieto y Olmos \& García, 2017) sitúan la digitalización de la educación como una apuesta emergente (Rivera-Vargas et al., 2018) y, sobre todo, necesaria, en unas escuelas que comienzan a construir sus oportunidades igualitarias de 
aprendizaje desde políticas (SITEAL, 2014; UNESCO, 2013) y prácticas tecnológicas (Arias y Cristia, 2014 y Pedró, 2017).

Probablemente, uno de los mayores elementos disruptivos en la literatura de referencia sobre la conceptualización de los centros educativos estriba en lo que se ha denominado como mobilelearning o aprendizaje móvil (Cobcroft, Towers, Smith \& Bruns, 2006; López- García, 2018; Torres, Infante \& Torres, 2015; UNESCO, 2013, 2017) por su capacidad de convertir la escuela en transformadora de su contexto desde el ámbito pedagógico. El aprendizaje móvil surge en estos momentos con una función socioeducativa, que ha sido abordada desde diferentes enfoques (Ramírez-Montoya y García-Peñalvo, 2017), pero también en distintos entornos (universitarios y no universitarios), en sistemas de aprendizaje a distancia (García-Aretio, 2017) y presenciales convencionales (Santiago, Tramaldo, Kamijo y Fernández, 2015), para áreas de conocimiento específicas (Cheung \& Slavin, 2013 y García \& Fombona, 2015 ) y/o como herramienta de formación docente (Fengchun, Mark, Hyo-Jeong \& Yancy, 2017), y en todos los casos con importantes aportaciones.

\section{c. Caracterización de las mejores escuelas desde la agenda política de la digitalización educativa}

Enmarcado en la "pedagogía electrónica" a la que hacen referencia Ballester y Colom (2011) y desde la que es posible contrarrestar gran parte de las críticas generadas por el uso negligente de los dispositivos móviles en las instituciones educativas (Beland \& Murphy, 2016; Englander, 2017), el aprendizaje móvil se impone como un instrumento principal en la consecución de la equidad educativa por parte de los centros. Así se constata en el discurso que ha venido desarrollando UNESCO en sus informes (UNESCO, 2012, 2013, 2017), cuyos planteamientos están incorporados en la agenda educativa global, pero con focos de interés muy distintos según quienes sean los actores políticos (Comisión Europea, OCDE entre otros).

UNESCO y Comisión Europea se han posicionado políticamente en materia de digitalización educativa y lo han hecho desde planos diferentes, aunque convergen en señalar el valor de estas políticas para el mercado laboral, la inclusión social y la mejora del sistema educativo. UNESCO desarrolla su discurso en torno a la democratización educativa, y contempla el aprendizaje móvil como una herramienta facilitadora de apertura, accesibilidad y ubicuidad de los procesos de aprendizaje. Sin ir más lejos, en el caso de la actual pandemia causada por el COVID-19, UNESCO está liderando la respuesta educativa mundial a través de tres líneas de acción (UNESCO, 2020a): (1) supervisión del impacto del COVID-19 en educación, (2) puesta en marcha de una comunidad para mejorar el intercambio de conocimientos, el aprendizaje entre iguales y la formación en aprendizaje abierto y a distancia, fomentando las alianzas público - privadas y (3) publicación de una lista de aplicaciones y plataformas de libre acceso para su uso por parte de la comunidad educativa (UNESCO, 2020b). Estas tres líneas de acción, asegura UNESCO, no deben perder de vista los principios de inclusión y equidad como principios rectores de la respuesta, en reconocimiento de que el cierre de escuelas tiende a afectar más a los alumnos vulnerables (UNESCO, 2020c).

La Comisión Europea por su parte, adopta una posición más economicista, defendiendo la digitalización educativa como un instrumento clave para el desarrollo económico y social de un país. Desde OCDE, no se plantean directrices sólidas de actuación y su estrategia es muy desigual, abarcando postulados que van desde el cuestionamiento de la digitalización y su vinculación con la mejora del rendimiento académico, hasta la defensa de esta en cuanto a mejora en el desarrollo de competencias globales. 
En este sentido, los principales estudios realizados en torno a esta temática se han centrado en evidenciar la potencialidad instrumental del aprendizaje móvil, siguiendo mayoritariamente directrices macropolíticas como el Plan de Acción de Educación Digital de la Comisión Europea de 2018, el Marco Estratégico del ET 2020, DigComp, DigCompEdu, DigCompOrg/SELFIE y Aprendizaje móvil de UNESCO.

Sin embargo, son escasos los trabajos preocupados por abordar las implicaciones de la digitalización en claves de igualdad y democratización educativa (Gros, 2012, 2015, 2019; Gros \& López, 2016), y no existe ninguna investigación que analice las coordenadas internacionales sobre las que sujetos políticos como UNESCO están construyendo la concepción de mejores escuelas en base al uso inclusivo que realizan del aprendizaje móvil.

El valor educativo de esta propuesta realizada por la UNESCO estriba en la legitimación que el aprendizaje móvil otorga a los centros para hacer de la gestión y organización de la educación un proyecto de gobierno escolar compartido por toda la comunidad. De este modo, el interés reside en destacar que desde la pluralidad de centros (Primaria y Secundaria), de contextos (países muy diferentes), desde la complejidad de su gestión y la variedad en su titularidad (privada, pública) todas las escuelas que participan entienden la importancia de compartir las tecnologías móviles, como la visión hacia la que dirigir sus esfuerzos y recursos. De ahí que como prácticas comunes en todos los centros analizados existen al menos estas tres directrices: un apoyo continuo al desarrollo profesional docente en torno a la digitalización, garantías en el equipamiento del alumnado y la conceptualización de la tecnología como instrumento educativo (de aprendizaje) y de gobierno.

\section{Objetivos del trabajo}

Partiendo de los estudios previos ya referidos, el trabajo que se presenta trata de radiografiar los componentes que sustentan las mejores prácticas de aprendizaje móvil que han sido seleccionadas por la UNESCO con la intención de aportar información sobre los contextos y los modelos de organización y gestión con los que se están construyendo los centros educativos elegidos. En este sentido, se pretende acotar los principios causales que subyacen en las mejores prácticas escolares tal y como son conceptuadas por actores de poder (UNESCO-Escuelas del Proyecto UNESCOFazheng) en el ámbito de la educación (Tabla 1).

Asimismo, el trabajo trata de plantear la idea de transferibilidad digital entre centros pertenecientes a países geográficamente muy heterogéneos (China, Rusia, España entre otros). El propósito es examinar las lecciones que pueden obtenerse del estudio comparado de estas escuelas.

Finalmente, hay una intención de estudiar si la caracterización que se está haciendo de las mejores escuelas a partir de las mejores prácticas de aprendizaje móvil, responde a patrones convencionales de evaluación, de digitalización de la educación o está asociada al discurso institucional que encierra la agenda política internacional (UNESCO). Desgranar la conceptualización de las mejores prácticas supone en realidad el inicio de una línea de investigación en torno al paradigma social del aprendizaje móvil (Pedró, 2017; Torres, Infante \& Torres, 2015; UNESCO, 2013, 2017, 2020b). De hecho, aunque muchas de las escuelas analizadas son públicas, es reseñable la función social que realizan estos centros cuyo proyecto está dirigido a colectivos muy heterogéneos, lo que hace que la diferenciación en cuanto a la titularidad, públicoprivado, deje de tener peso importante para centrarse en componentes que apuntan a los principios de equidad. 
Para responder a estas cuestiones, el artículo se ha centrado en la estrategia bottom-up (tabla 1) porque se entiende que la transformación educativa genuina podrá alcanzarse en la medida en que las dinámicas de trabajo contemplen tácticas que surjan de lo local y alcancen una dimensión global. Se sugiere, por lo tanto, la idea de que los equipos directivos y docentes implicados en el día a día de las escuelas, junto con familias, comunidad educativa y otros grupos interesados (asociaciones profesionales, sindicatos, fundaciones grupos empresariales, etc.), son los agentes que, conociendo las necesidades de su entorno inmediato, están especialmente motivados y contextualmente capacitados para diseñar y desarrollar proyectos orientados a la disminución de la inequidad y la mejora de la excelencia educativa.

\begin{tabular}{|llll|}
\hline País & Centro educativo & Titularidad & $\begin{array}{l}\text { Inicio de los } \\
\text { proyectos }\end{array}$ \\
\hline Brasil & Colégio Miguel de Cervantes & Privada & 2012 \\
\hline China & $\begin{array}{l}\text { Beijing Royal School } \\
\text { Shuren Jingrui Primary School }\end{array}$ & $\begin{array}{l}\text { Privada } \\
\text { Pública }\end{array}$ & $\begin{array}{l}2010 \\
2010\end{array}$ \\
\hline España & CEIP Ponte dos Brozos & Pública & 2012 \\
\hline Israel & Amal Shevach Mofet High School & Privada & 2013 \\
\hline Portugal & Colégio Monte Flor & Privada & 2013 \\
\hline Reino Unido & Denbigh High School & Pública & 2013 \\
\hline \multirow{2}{*}{ Rusia } & The International Educational Complex "Harmony & Pública & 2012 \\
\hline
\end{tabular}

Tabla 1. Prácticas escolares exitosas de aprendizaje móvil

Fuente: Elaboración propia a partir de UNESCO (2018)

\section{Material y métodos}

Metodológicamente este trabajo se ha diseñado desde el análisis político del discurso recogido por Rodríguez Fernández (2018) y combinado con un estudio de casos a partir del cotejo de documentos y materiales del Proyecto UNESCO - Fazheng (UNESCO, 2018). El modelo propuesto se engloba en un diseño de investigación más amplio que está siendo utilizado en otros trabajos (Muñoz Rodríguez et al., 2020) y que se ha elaborado con el propósito de examinar las continuidades-discontinuidades de los discursos de Organismos Internacionales en materia de digitalización educativa. En el presente estudio se aborda el papel de la UNESCO a través de las prácticas de aprendizaje móvil seleccionadas. De forma específica, el enfoque utilizado nos permite abordar la construcción del éxito educativo a partir las identidades discursivas de actores políticos como la UNESCO. EI material utilizado como objeto de estudio está constituido por las 7 mejores prácticas de aprendizaje móvil que han sido seleccionadas por la UNESCO (de entre más de 150 candidaturas de 41 países diferentes). Los descriptores empleados para el análisis de los campos temáticos se han formulado a partir de los criterios de elegibilidad de las mejores escuelas utilizados por UNESCO (Figura 1). De este modo, partiendo de los 8 ejes de análisis (visión, planificación, entorno del aprendizaje móvil, estrategia de fortalecimiento, mejores prácticas y logros, logros globales, sostenibilidad, innovaciones) que contempla UNESCO y que vendrían a caracterizar la dimensión institucional de su discurso con relación a cómo se construyen los mejores centros, en el presente trabajo se han realizado adaptaciones estructurales, que no afectan al contenido, y que nos permiten mostrar las claves en las están representadas las 
escuelas transformadoras de todo el mundo (ya sean claves de excelencia educativa, de digitalización educativa, y/o siguiendo la agenda política de la digitalización).

Es pertinente destacar la existencia de un sesgo en la selección de las escuelas -puesto que el artículo se remite a escuelas del Proyecto UNESCO-Fazheng (UNESCO, 2018)- que ha sido utilizado para formular el estudio desde los principios del análisis sociopolítico y crítico del discurso establecido por Laclau (2005). UNESCO, como se ha señalado con anterioridad, abrió una convocatoria de propuestas dirigida a instituciones que reuniesen los siguientes criterios de elegibilidad: (a) representan una escuela individual o un grupo de escuelas; (b) cuentan con un modelo de aprendizaje móvil que abarca toda la escuela - una serie de prácticas de aprendizaje móvil para el aprendizaje, la enseñanza y la gestión escolar - que ya se ha aplicado; (c) imparten enseñanza primaria y/o secundaria; (d) el modelo de aprendizaje móvil se está aplicando en distintos grados y asignaturas de la escuela; y (e) dispone de pruebas (basadas en datos y otras pruebas confirmadas) que demuestran que el modelo de aprendizaje móvil ha resultado eficaz.

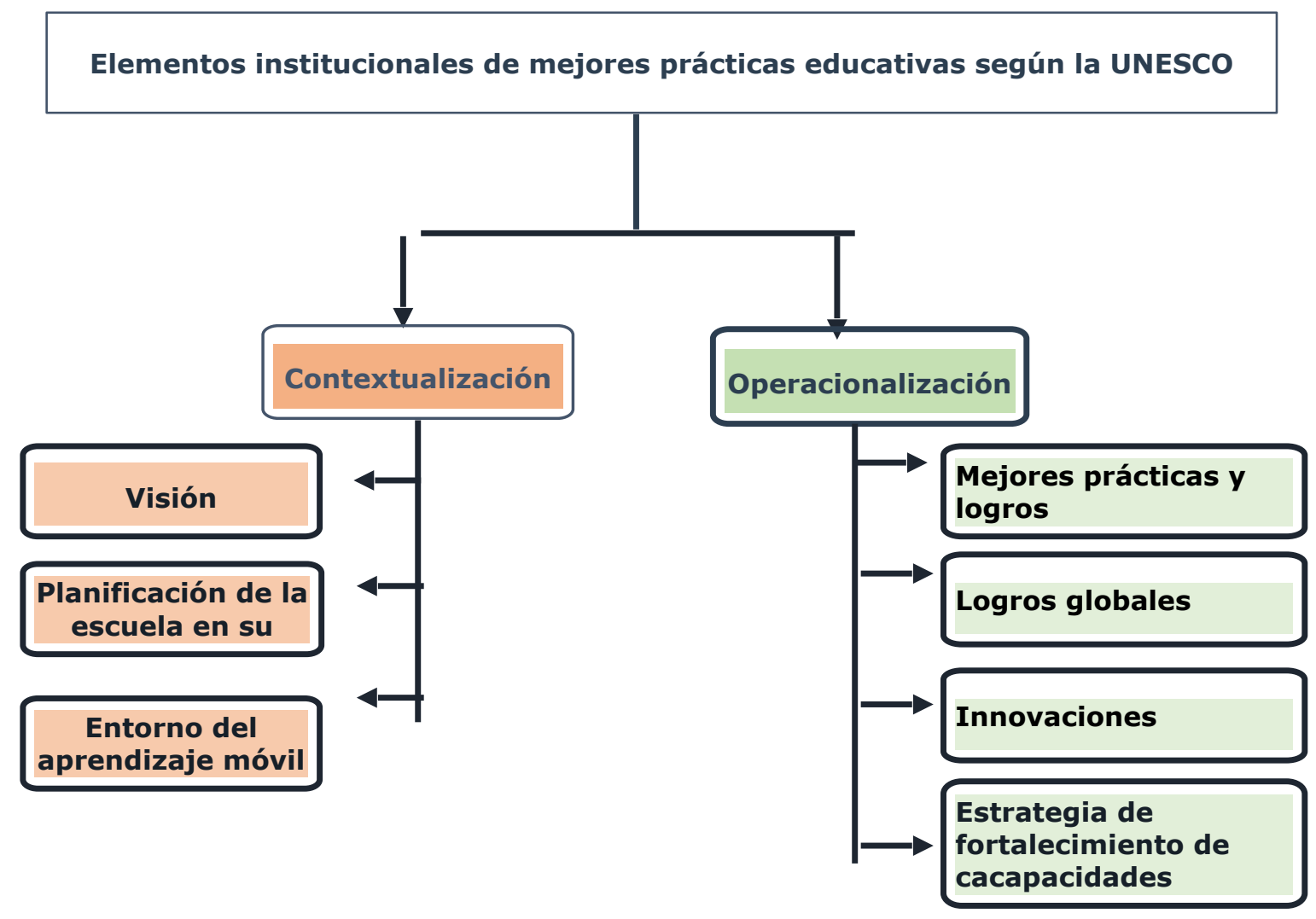

Figura 1. Criterios de elegibilidad de las mejores escuelas.

Fuente Elaboración propia

Desde el análisis de los niveles internos del enunciado se han introducido algunas variantes que han (re)articulado las categorías iniciales. Así, siguiendo la dimensión de la construcción hegemónica del discurso (Fair, 2019) se han estructurado las unidades de registro (Aigneren, 2009) en tres categorías (modelo contextual, entorno del aprendizaje móvil e impacto y transferibilidad) creadas en el marco del análisis del discurso político (Fair, 2019) que aglutinan los elementos lingüísticos "de lo que se enuncia" en las escuelas que han sido seleccionadas y "de cómo se enuncia" en los documentos de la UNESCO. En ambos casos, la contextualización se asienta sobre 
componentes de identidad explicativos de las estrategias de intervención y los modelos operativos seleccionados, siguiendo las claves políticas de caracterización de las mejores prácticas (Figura 2).

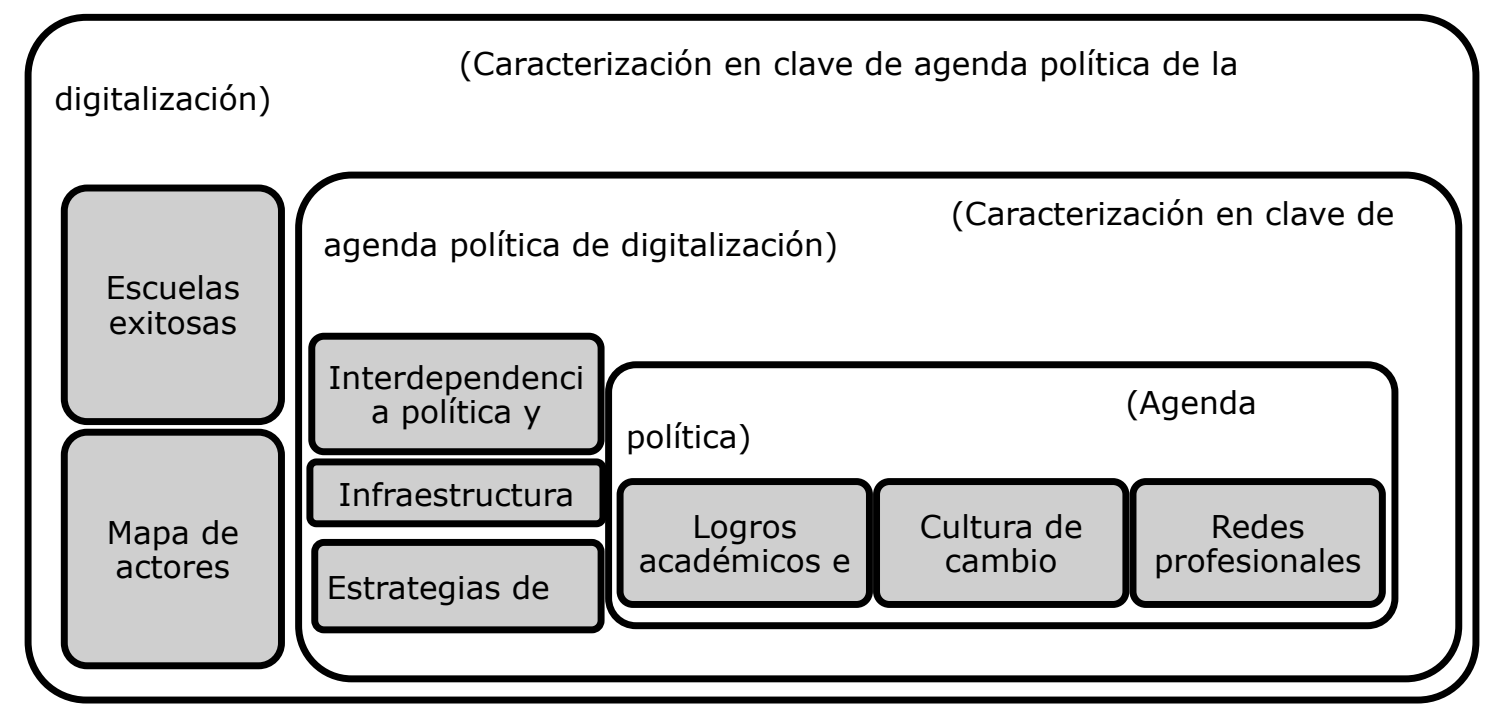

Figura 2. Categorías de análisis Fuente: Elaboración propia

En las categorías de análisis propuestas se ha tratado de establecer una analogía entre unidades de estudio y herramientas del discurso político. De ahí que el entorno de aprendizaje móvil aparezca como un bloque de análisis con autonomía con respecto a los criterios propuestos por la UNESCO.

En este sentido, la primera categoría que hemos denominado "Modelo contextual" se corresponde con las identidades del primer nivel de análisis del discurso político y trata de filtrar información con la que se identifican las mejores escuelas. Para ello los descriptores utilizados han sido dos: la caracterización de las escuelas exitosas y el mapa de actores en el aprendizaje móvil.

La segunda categoría se construye desde los parámetros marcados por el enriquecimiento de las oportunidades de aprendizaje que pueden brindar las tecnologías, y desde los contextos que sirven para la producción de estos entornos de aprendizaje virtual. En el marco del análisis del discurso alude a los elementos problematizados por sus agentes. La interdependencia con marcos políticos y correlación pedagógica, la infraestructura tecnológica (y las licencias) y las estrategias de fortalecimiento son los tres descriptores identificados.

La tercera categoría representa lo que Fair (2019) denomina significantes legitimadores, y que en el trabajo hemos referido a los valores a los que la UNESCO le otorga una significación notoria (logros académicos e inclusivos, cultura de cambio y redes profesionales). La transferibilidad de las mejores prácticas se asienta en tres descriptores significativos: logros académicos, cultura de cambio y redes profesionales, inferidos del discurso utilizado por la UNESCO para resaltar principalmente la forma en la que se presenta su discurso (logros académicos e inclusivos, cultura de cambio y redes profesionales). El propósito no es otro que servir de análisis para el estudio de la transferibilidad de las que han sido conceptuadas como las mejores escuelas. 


\section{Resultados}

Las tres categorías analizadas nos han permitido conocer los parámetros (de excelencia, de digitalización y/o de agenda política de la digitalización) sobre los que se están construyendo las mejores prácticas educativas, así como los modelos contextuales, de gestión y los patrones educativos que exportan a otros países, tal y como se recoge en la comparación realizada por apartados. Se ha tratado igualmente de responder a los escenarios de futuro atendiendo a sinergias educativas (centros escolares transformadores) y a políticas internacionales (UNESCO). Más allá de discursos formales generados en el marco de la pandemia de la COVID-19 y que se han visto alentados con el uso generalizado de las tecnologías a raíz de la suspensión de la actividad académica (recientemente acaba de hacerse pública por parte de la "Campaña por una Infancia sin comerciales" y la "Red de acción del tiempo que los niños pasan en una pantalla" una Declaración sobre las Tecnologías Aplicadas a la Educación y las políticas educativas durante la pandemia 11 de agosto de 2020), el trabajo que se presenta se construye desde la naturaleza pedagógica de la digitalización educativa y desde los valores de inclusión que conforman las políticas educativas institucionales (UNESCO).

De manera general, el estudio de los descriptores relativos a la primera categoría de análisis (escuelas exitosas y mapa de actores) ha evidenciado que se está fraguando una narrativa de las mejores prácticas educativas a partir de la caracterización digital y política (agenda global) de los centros escolares. En ese sentido, los elementos de titularidad (público-privada y el principio de la libertad de elección) y los indicadores de rendición de cuentas (autonomía de mercado) reconocidos hasta ahora como instrumentos legítimos del gobierno de los centros educativos, y vinculados a un modelo de escuelas eficaces y exitosas (Darling-Hammond et al., 2017; Delannoy \& Guzmán, 2009 y OCDE, 2016) pierden un protagonismo que comienza a ser asumido por la digitalización educativa. Del análisis de este primer bloque se puede deducir que el aprendizaje móvil ha pasado de ser concebido como parte de una metodología innovadora a convertirse en un referente contextual. Así, es constatable que la función social e inclusiva que desempeñan estos centros a través de su proyecto de aprendizaje móvil comienza a desdibujar el criterio selectivo de los modelos de excelencia educativa, haciendo que el foco recaiga en cuestiones puramente educativas y no tanto en la naturaleza público-privada de los mismos.

El estudio de la segunda categoría relativa a la caracterización del entorno de aprendizaje ha contribuido a identificar la hegemonía de las interacciones a diferentes escalas en la configuración programática del aprendizaje, poniendo de manifiesto la necesidad de operativizar las alianzas estratégicas relativas a las licencias tecnológicas que comienzan a entenderse como un factor de oportunidades.

En cuanto a la tercera categoría (impacto y transferibilidad), el trabajo realizado pone de manifiesto que las lecciones aprendidas entre contextos geoterritoriales diversos (como los que son objeto de estudio en la presente investigación), están centrados en resaltar los elementos comunes que resultan del paradigma del aprendizaje móvil y que tienen que ver con los parámetros de éxito educativo y de desarrollo social e inclusivo, sobre los que se orientan las instituciones educativas analizadas.

\section{a. Modelos contextuales y caracterización de las mejores prácticas.}

Las escuelas seleccionadas por UNESCO como prácticas escolares de aprendizaje móvil exitosas mantienen convergencias y divergencias en los dos descriptores que tienen que ver con los modelos contextuales: la caracterización del centro y el mapa de actores involucrados en los procesos de aprendizaje móvil. El análisis de los descriptores utilizados en esta categoría muestra varios elementos de interés, tal y como se evidencia en las tablas 2 y 3. 
El estudio comparativo de los modelos de escuelas exitosas permite extraer algunas ideas interesantes. De un lado, el estudio comparativo del descriptivo de caracterización del centro (Tabla 2) evidencia, en primer lugar, que la ratio alumnos/profesor baja en los centros privados (salvo en el caso del Harmony School que tiene una ratio de 10 alumnos por profesor). En segundo lugar, son los centros públicos los que mayor diversidad en el entorno sociocultural presentan (salvo el Amal Shevach Mofet en Israel que tiene familias de todos los estratos de la población). Por lo general estas valoraciones concuerdan con el estado general de los sistemas educativos en los países desarrollados, pero es en las excepciones donde está el mayor interés. Tanto el Harmony School ruso (público) como el Amal Shevach Mofet israelí (privado) consiguen salirse de la norma y lograr prácticas exitosas siendo centros con una ratio baja y amplia diversidad en el perfil sociocultural de sus alumnos.

\begin{tabular}{|c|c|c|c|c|}
\hline & Etapas & Alumnos & Docentes & Perfil sociocultural \\
\hline $\begin{array}{l}\text { Beijing Royal } \\
\text { School }\end{array}$ & Secundaria & 950 & $x$ & Alto \\
\hline $\begin{array}{l}\text { Shuren Jingrui } \\
\text { Primary School }\end{array}$ & Primaria & 1400 & 67 & $\begin{array}{l}>1 \% \text { licenciatura o superior } \\
65 \% \text { trabajadores manuales } \\
\text { o agricultores }\end{array}$ \\
\hline $\begin{array}{l}\text { Amal Shevach } \\
\text { Mofet }\end{array}$ & Secundaria & 900 & 70 & $\begin{array}{l}\text { Todos los estratos de la } \\
\text { población }\end{array}$ \\
\hline Colégio Monte Flor & Primaria & $x$ & $x$ & $\begin{array}{l}\text { Urbano } \\
\text { Alto }\end{array}$ \\
\hline Harmony School & $\begin{array}{l}\text { Infantil, } \\
\text { Primaria y } \\
\text { Secundaria }\end{array}$ & 2000 & 200 & $\begin{array}{l}\text { Amplia gama de entornos } \\
\text { culturales diferentes }\end{array}$ \\
\hline $\begin{array}{l}\text { CEIP Ponte dos } \\
\text { Brozos }\end{array}$ & $\begin{array}{l}\text { Infantil y } \\
\text { Primaria }\end{array}$ & 1100 & 80 & $\begin{array}{l}\text { Población joven } \\
46 \text { nacionalidades }\end{array}$ \\
\hline $\begin{array}{l}\text { Denbigh High } \\
\text { School }\end{array}$ & Secundaria & 1120 & $x$ & $\begin{array}{l}\text { Áreas y comunidades más } \\
\text { desfavorecidas de la región }\end{array}$ \\
\hline $\begin{array}{l}\text { Colégio Miguel de } \\
\text { Cervantes }\end{array}$ & $\begin{array}{l}\text { Infantil, } \\
\text { Primaria y } \\
\text { Secundaria }\end{array}$ & 1500 & 152 & Alto \\
\hline
\end{tabular}

Tabla 2. Caracterización de las escuelas exitosas

Fuente: Elaboración propia

De otro lado, la comparación del descriptor de actores (Tabla 3) evidencia algunos aspectos destacables. En primer lugar, el compromiso de la comunidad educativa es un requisito sine qua non para el éxito del proyecto. La participación de entidades públicas o privadas se valora por parte de UNESCO. En segundo lugar, el ejemplo del centro público ruso y el centro privado portugués evidencian que la titularidad del centro no condiciona la implicación de actores externos, ni define el tipo de actor (empresa, administración pública u organismo internacional) con el que colaboran. En tercer lugar, los centros con perfiles socioculturales bajos o muy diversos, tienen más dificultades para tejer redes externas (salvo el caso del centro ruso). 


\begin{tabular}{|c|c|c|c|c|c|c|c|c|c|c|}
\hline & $\begin{array}{l}\text { Eq. } \\
\text { Direc } \\
\text { tivo }\end{array}$ & $\begin{array}{l}\text { Doc } \\
\text { ent } \\
\text { es }\end{array}$ & $\begin{array}{l}\text { Fa } \\
\text { mili } \\
\text { as }\end{array}$ & $\begin{array}{l}\text { Pers } \\
\text { onal } \\
\text { de } \\
\text { Admi } \\
\text { nistr } \\
\text { ación } \\
\text { y } \\
\text { Servi } \\
\text { cios }\end{array}$ & $\begin{array}{l}\text { Em } \\
\text { pre } \\
\text { sas }\end{array}$ & $\begin{array}{l}\text { Org } \\
\text { ani } \\
\text { sm } \\
\text { os } \\
\text { Int } \\
\text { ern } \\
\text { aci } \\
\text { ona } \\
\text { les }\end{array}$ & $\begin{array}{l}\text { Red } \\
\text { es } \\
\text { ext } \\
\text { ern } \\
\text { as }\end{array}$ & $\begin{array}{l}\text { Fun } \\
\text { dac } \\
\text { ion } \\
\text { es }\end{array}$ & $\begin{array}{l}\text { Univ } \\
\text { ersid } \\
\text { ades }\end{array}$ & $\begin{array}{l}\text { Ad. } \\
\text { Públ } \\
\text { ica }\end{array}$ \\
\hline $\begin{array}{l}\text { Beijing Royal } \\
\text { School }\end{array}$ & $X$ & $x$ & & & & $\mathrm{X}$ & & & & \\
\hline $\begin{array}{l}\text { Shuren Jingrui } \\
\text { Primary School }\end{array}$ & $x$ & $x$ & & $x$ & & & & & & \\
\hline $\begin{array}{l}\text { Amal Shevach } \\
\text { Mofet }\end{array}$ & $x$ & $x$ & & $x$ & & & $x$ & & & \\
\hline Colégio Monte Flor & $x$ & $x$ & $x$ & & $x$ & & & & $x$ & $x$ \\
\hline Harmony School & $\mathrm{X}$ & $X$ & $X$ & $\mathrm{X}$ & $x$ & $\mathrm{X}$ & $x$ & & & \\
\hline $\begin{array}{l}\text { CEIP Ponte dos } \\
\text { Brozos }\end{array}$ & $\mathrm{X}$ & $\mathrm{X}$ & & & & & & $x$ & & $x$ \\
\hline $\begin{array}{l}\text { Denbigh High } \\
\text { School }\end{array}$ & $x$ & $x$ & & $x$ & & & & & & \\
\hline $\begin{array}{l}\text { Colégio Miguel de } \\
\text { Cervantes }\end{array}$ & $x$ & $x$ & & $x$ & & & & & & $x$ \\
\hline
\end{tabular}

Tabla 3. Mapa de actores implicados

Fuente: Elaboración propia

\section{b. Entornos de aprendizaje}

Todas las escuelas seleccionadas han articulado entornos de aprendizaje móvil (figura 3) bajo el marco conceptual de "educación para la ciudadanía global" que subyace en la UNESCO. Igualmente, las propuestas están basadas en el modelo de aprendizaje personalizado e inclusivo, a partir del cual se coordinan, movilizan y comparten recursos, alineado con el enfoque "Bring your own device" (BYOD), "trae tu propio dispositivo".

Los contextos donde se asientan estos ambientes de aprendizaje están definidos por las relaciones de interdependencia con marcos políticos y de correlación pedagógica con el plan de estudios de los propios centros. Las iniciativas están vinculadas en algunos casos a los respectivos Ministerios de Educación de los países que actúan como motores de impulso (como por ejemplo en las escuelas de Brasil, Portugal e Israel) en las mismas. A ello, se le suma la participación (especialmente con cobertura financiera) de fundaciones y grupos privados (Colegio Monte Flor de Portugal y CEIP Ponte dos de España) que han contribuido a la expansión y consolidación de los proyectos. De la misma manera, estas relaciones han llegado a alcanzar otras esferas que han permitido la incorporación de agentes externos como universidades extranjeras (Beijing Royal, China), empresas tecnológicas (Colegio Monte Flor de Portugal) y trabajadores privados que han participado en el desarrollo profesional de los docentes (CEIP Ponte dos de España).

Al mismo tiempo, podemos hablar de la existencia de corresponsabilidad programática en estos entornos virtuales ya que las actuaciones están en consonancia con los planes de estudio en general y con determinados proyectos educativos en particular $y$, siempre derivados del enfoque 
pedagógico del centro que suele ser multidisciplinar. Así pues, las experiencias abarcan todas las áreas del plan de estudios y todos los niveles salvo en aquellos casos donde las escuelas sólo cubren determinados grados educativos.

Otro de los descriptores identificados atiende a la necesidad de configurar una infraestructura tecnológica sólida para la ejecución y desarrollo de los diferentes proyectos educativos. La dotación de equipamiento, que abarca a una amplia gama de dispositivos digitales, conlleva la adquisición de licencias de tecnología en colaboración con empresas del sector, que se extiende incluso a cuestiones de seguridad electrónica. Ello hace que se cuente con unos sistemas robustos en cuanto a infraestructura, salvo en el caso de la escuela de Israel que manifiesta no disponer de mucha especialización en cuanto a recursos.

En este sentido, llama la atención la presencia de iniciativas que, en escuelas como la española, utilizan contenidos provenientes de una plataforma educativa privada cuando, la mayoría de ellas, se nutren de contenidos públicos proporcionados por plataformas educativas estatales (China y Rusia). Igualmente, destacable es el caso de la escuela Amal Shevach Mofet, en Israel, que forma parte de una potente red educativa, y de la escuela inglesa (Denbigh) que se nutre de intercambio público de mejores prácticas.

En todas las escuelas se cuenta con soporte educativo propio y se anima a profesores, alumnos e incluso a las familias (caso de Shuren Jingrui, China) a crear sus propios materiales, con el propósito de poder compartirlos y generar bases de recursos utilizadas por los centros y los usuarios.

Finalmente, el tercer apéndice de esta categoría recaería en las estrategias de fortalecimiento. Identificamos entre ellas, el compromiso general asumido en cuanto a la necesidad de capacitar al profesorado para garantizar que los docentes dominen los recursos e instrumentos del entorno digital. La formación continua, el reclutamiento de docentes cualificados, el estudio de buenas prácticas, las auditorias y el liderazgo escolar participativo, han sido algunas de las prácticas que han contribuido a dotar de robustez a los proyectos. En la misma línea, cabe destacar las medidas incentivadoras que se han implementado y que recaen principalmente en el profesor. Así pues, los premios, y las becas se otorgan por creación de material educativo digital y, esta producción se contempla en las evaluaciones anuales de desempeño (Jingrui y Beijing Royal, China). 


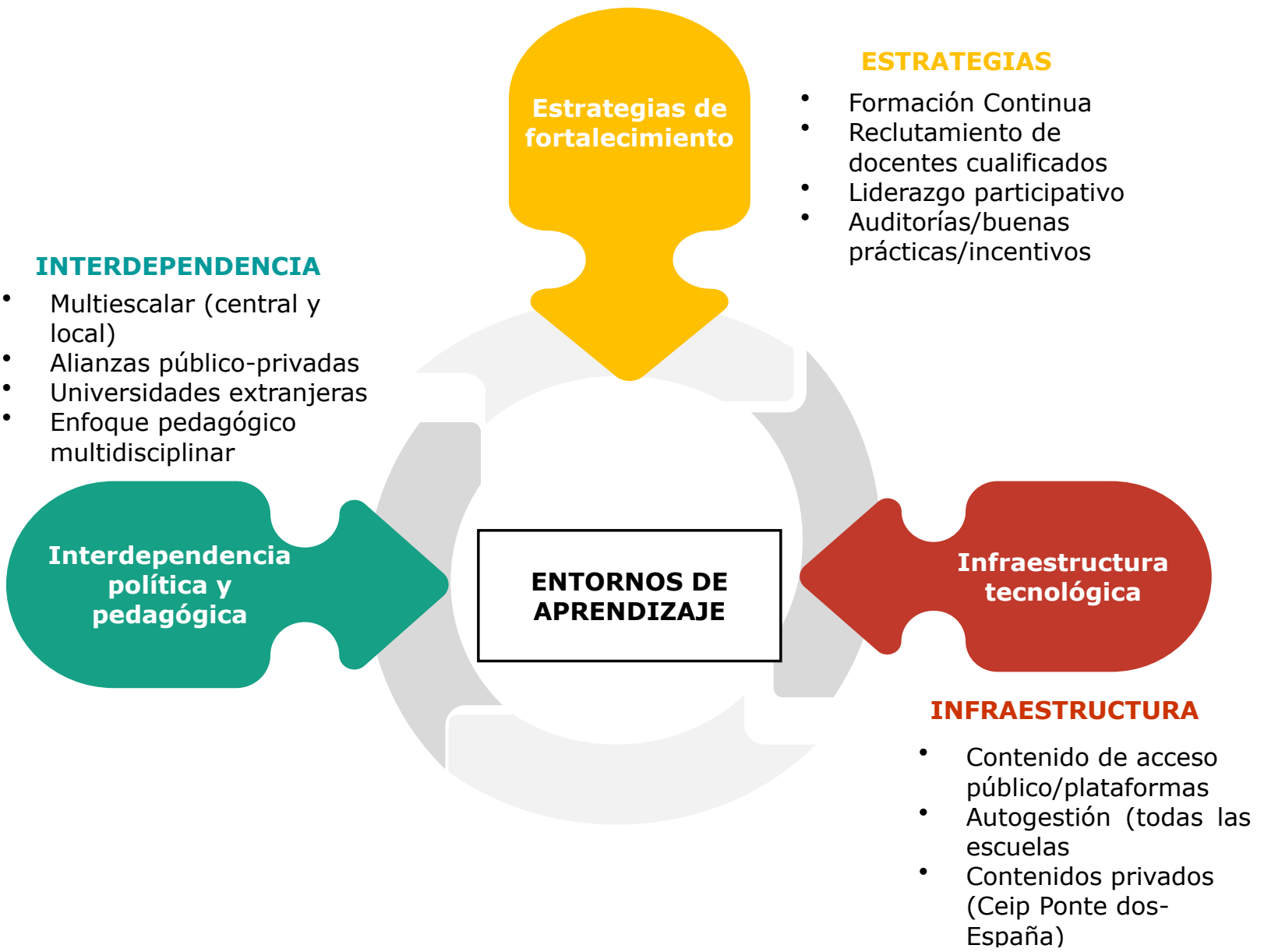

Figura 3. Entornos de aprendizaje: la convergencia política y educativa.

Fuente: Elaboración propia

\section{c. Impacto y transferibilidad}

Los tres descriptores recogidos en esta categoría: logros académicos e inclusivos, cultura de cambio y la creación de redes profesionales, muestran varios elementos de interés, tal y como se evidencia en la figura 4, en la que se han ordenado las experiencias por nivel de significatividad en los tres objetos de análisis.

\section{Logros académicos e inclusivos:}

De un lado, el estudio comparativo constata la existencia de una correspondencia entre la cultura colaborativa por parte de todos los miembros de la comunidad educativa (profesorado, alumnado, padres, comunidad local y administración escolar) y los logros obtenidos tanto en resultados académicos, como en el desarrollo de proyectos de digitalización educativa. Se muestra así que la responsabilidad compartida (liderazgo compartido) es una de las claves de modelos educativos que destacan por sus altos logros.

En términos de impacto académico e inclusivo, la idea que se trasmite es que la gestión y la organización de la educación en torno a un proyecto de aprendizaje móvil con colectivos de población muy heterogéneos es perfectamente convergente con el lenguaje de estándares nacionales e internacionales (es el caso por ejemplo de Denbigh High School de Reino Unido, de la 
Shuren Jingrui Primary School de China- donde se trabaja en entorno rural y marginal- y de la escuela Harmony School rusa.

En cambio, en otras escuelas como la Amal Shevach Mofet de Israel, el Colegio Monte Flor de Portugal, y el Colegio Miguel de Cervantes de Brasil, los logros principalmente se han producido en el plano de la inclusión, pero queda pendiente equiparar la vertiente académica, dimensión esta que se ha conseguido en términos de competencias cognitivas y motivación en el CEIP Ponte dos de España.

De todos modos, la idea de globalidad queda recogida en el proyecto de ciudadanía holístico que se plantea en la Beijing Royal de China, donde el progreso académico se entiende desde la multiculturalidad y se realiza con el aprendizaje móvil.

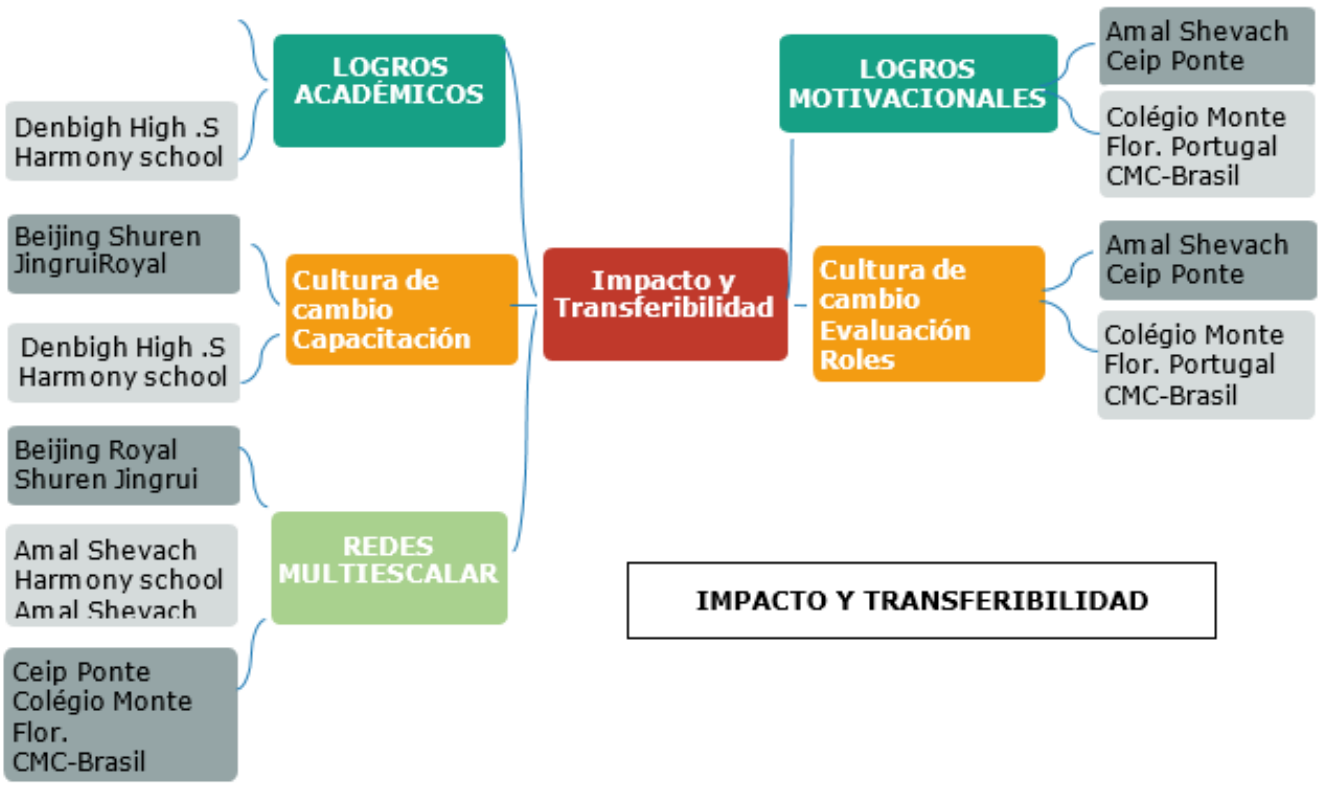

Figura 4. Identidad y proyección de las mejores prácticas (excelencia/digitalización/agenda política). Fuente: Elaboración propia

\section{Cultura de cambio:}

Por otra parte, el análisis de la cultura de cambio es un valor que es compartido de manera general por todas las escuelas seleccionadas por la UNESCO. La idea de la flexibilidad como estrategia de mejora es una constante en la identidad institucional de centros transformadores que permite una mejora permanente (así se pone de manifiesto en el Denbigh High School de Reino Unido, la Amal Shevach Mofet de Israel, en la Harmony School rusa a través de sus proyectos de desarrollo a nivel de centro). El caso de la Shuren Jingrui Primary School de China es reseñable porque muestra las fases de construcción interna de su proyecto de aprendizaje móvil desde la implicación de los miembros del centro, siendo el centro en el que mayores han sido los cambios que se han 
producido. Queda pendiente, no obstante, un trabajo de coordinación en la transición a los centros de educación secundaria.

El Colegio Monte Flor de Portugal, el Colégio Miguel de Cervantes de Brasil y el CEIP Ponte dos de España expresan el capital educativo que supone el proyecto digital para la mejora de la colaboración docente. La digitalización del aprendizaje móvil se convierte en estos casos en el hilo conductor de prácticas más democráticas y coordinadas en la formación de profesores, así como en la articulación de políticas pedagógicas más inclusivas.

De nuevo es la Beijing Royal (China) la escuela que realiza un planteamiento global desde la idea de los currículos internacionales y su relación con diferentes sistemas de calificación (EEUU, Canadá).

\section{Creación de redes profesionales:}

Junto a los descriptores anteriores (logros académicos e inclusivos/cultura de cambio) hemos incluido otro elemento (creación de redes profesionales) que está muy presente en esta categoría y que evidencia el impacto que el modelo de aprendizaje móvil tiene a partir de su exportación a otros centros educativos de la misma ciudad, o de otras ciudades y otros ámbitos territoriales. Este impacto es cuantificado por las transferencias que se producen y que en muchos casos se traduce en un mayor reconocimiento profesional por el desempeño docente realizado.

Son destacables, por su capacidad de transferibilidad, la escuela de Rusia cuyo modelo de aprendizaje móvil ha convertido al centro en una Escuela de Educación a distancia para otros centros. Probablemente el caso más extraordinario está representado por la Escuela de Educación Primaria de China que ha pasado de importar conocimiento sobre aprendizaje móvil a ser exportadora de su proyecto a escuelas situadas en otros países, como: Beijing, Shanghai, Guangzhou. En el caso de China hay una interdependencia institucional (a nivel gubernamental, empresarial y de centro) que permite la implementación con éxito (campus inteligente, uso gratuito de plataforma, etc) de este modelo de aprendizaje y su transformación educativa y social. Mientras que la escuela la Beijing Royal (China) internacionaliza su proyecto pedagógico a partir del reclutamiento de profesores de diferentes países en función de su grado de competencia en tecnologías educativas.

En definitiva, recogiendo una de las líneas comunes a las tres categorías de análisis, podemos considerar que no sería posible extraer un único modelo de centro exitoso para UNESCO, sino que su selección pone en evidencia que la diversidad de entornos y contextos no son limitantes para un desarrollo exitoso del aprendizaje móvil. El único requisito podría encontrarse en el compromiso de la comunidad educativa y en la conveniencia de tejer redes con entidades públicas o privadas que den una dimensión internacional a la digitalización educativa. Abordaremos esta y otras ideas que se pueden extraer de la discusión de los resultados en el siguiente apartado de conclusiones.

\section{Discusión y conclusión}

El aprendizaje móvil asume el doble reto planteado por una educación inclusiva y de calidad. Las prácticas premiadas por el Proyecto UNESCO-Fazheng ponen en evidencia, como se ha comprobado, la necesidad de repensar la evaluación de las prácticas escolares. Como ha puesto de relieve la crisis del coronavirus, se hace necesario incorporar esa dimensión social de inclusión, más allá de una dimensión formal y estandarizada del éxito educativo limitado a la excelencia académica. 
En las prácticas analizadas, la diversidad en el perfil sociocultural de los alumnos no es obstáculo para acometer transformaciones de este calado. Además, es muy probable que no exista un modelo único de centro exitoso para la UNESCO, ya que la selección realizada pone en evidencia que la diversidad de entornos no limita la implantación eficaz del aprendizaje móvil. Eso revela que desde una perspectiva geopolítica y a escala internacional, un requisito fundamental podría encontrarse precisamente en el compromiso de implicar a toda la comunidad educativa, tejiendo redes con entidades públicas y privadas, incorporando esa dimensión global a la digitalización educativa.

En este sentido, cabe destacar la función directiva y el compromiso docente como componentes básicos de ese éxito, que deben orientarse a calibrar adecuadamente el uso y el provecho de las herramientas para reducir la brecha digital, además de promover pedagogías de carácter colaborativo, reflexivo y de indagación, propias de proyectos de talante cooperativo donde lo digital adquiere una relevancia destacada. Si la tecnología digital ofrece una puerta abierta a un inmenso número de recursos, es cierto también que puede incrementar el riesgo de infoxicación y de pérdida de calidad democrática de los aprendizajes: resulta fundamental que una educación digital se oriente más a proporcionar y reforzar criterios que a sumar contenidos.

Asimismo, el análisis de las prácticas educativas seleccionadas nos ha permitido constatar que el aprendizaje móvil se está convirtiendo en un nuevo paradigma educativo integrador de tendencias divergentes, capaz de incorporar en un mismo modelo la eficacia de los sistemas excelentes de aprendizaje (excelencia educativa) con metodologías innovadoras (innovación tecnológica) y desde parámetros de inclusión y justicia social (políticas públicas de digitalización educativa).

El trabajo realizado subraya la necesidad de repensar la autonomía de los centros educativos a partir de esta línea de investigación que muestra que el aprendizaje móvil puede ser un elemento legitimador del gobierno de los centros docentes. Las tecnologías digitales, como se ha venido comprobando en los casos estudiados, intentan superar las clásicas restricciones de orden estructural que suelen presentar las escuelas tradicionales, limitaciones tanto de orden físico, relativas a la distribución de espacios, como de orden simbólico. La vinculación de estas prácticas digitales desde un modelo de gestión con más capacidad de decisión de los centros docentes supone un reto para los sistemas educativos y una oportunidad para alcanzar una educación inclusiva y de calidad.

\section{Reconocimientos}

Este trabajo está financiado por el proyecto nacional del Ministerio de Ciencia, Innovación y Universidades: "La identidad hiperconectada de la juventud y su percepción del tiempo en el ocio digital", con referencia PGC2018-097884-B-I00; y por el Proyecto regional de la Junta de Castilla y León: Identidades digitales en jóvenes hiperconectados: retos para el contexto familiar, social y escolar, con referencia SA038G19.

\section{Referencias}

Aigneren, M. (2009). Análisis de Contenido. Una Introducción. La Sociología en sus Escenarios, 1-52. Recuperado de: https://revistas.udea.edu.co/index.php/ceo/article/view/1550

Araujo C.F., Dias, E.J. \& Ota, M.A. (2014). The Tablet Motivating Mathematics Learning in High School. In M. Kalz, Y. Bayyurt, \& M. Specht (Eds.), Mobile as Mainstream - Towards Future Challenges in Mobile Learning (pp. 42-51). Springer International Publishing. https://doi.org/10.1007/978-3-319-13416-1 
Area, M.,Santana, P.J y Sanabria, A.N. (2020). La transformación digital de los centros escolares. Obstáculos y resistencias. Digital Education Review, 37, 15-31. https://doi.org/10.1344/der.2020.37.15-31

Arias Ortiz, E., y Cristia, J. N. (2014). El BID y la tecnología para mejorar el aprendizaje: ¿cómo promover programas efectivos? Banco Interamericano de Desarrollo. Recuperado de: https://bit.ly/2zxTqfi

Ball, S., y Youdell, D. (2007). Privatización encubierta de la educación. Informe Preliminar de $V$ Congreso Mundial de la Educación. Instituto de Educación, Universidad de Londres. Recuperado de: https://bit.ly/ 3aHiqOg

Ballester, L.I y. Colom, A.J. (2011). Digitalización y Tecnología Educativa, Revista portuguesa de pedagogía. Número Extra, 95-112. https://doi.org/10.14195/1647-8614_Extra-2011_8

Beland, L.P. \& Murphy, R. (2016). Ill communication: Technology, distraction, \& student performance. Labour Economics, 41, 61-76. https://doi.org/10.1016/j.labeco.2016.04.004

Brazuelo, G.F., Gallego, D.J y Cacheiro, M.L. (2017). Los docentes ante la integración educativa del teléfono móvil en el aula. RED. Revista de Educación a Distancia, 52(6) ,1-22. http://dx.doi.org/10.6018/red/ $\underline{52 / 6}$

Burbules, N. C. (2014). El aprendizaje ubicuo: nuevos contextos, nuevos procesos. Entramados: educación y sociedad, 1(1), 131-134. Recuperado de: https://bit.ly/2xomiq4

Caldeiro, M.C., Yot, C. y Castro, A. (2018). Detección de buenas prácticas docentes de uso de dispositivos móviles en primaria a través del análisis documental. Prisma social, 20, 58-75. Recuperado de: https:// bit.ly/2yVAUNM

CCFC (2020). Declaración sobre las tecnologías aplicadas a la educación y las políticas educativas durante la pandemia https://acortar.link/bU4Ae

Casassús, J. (2010). Las Reformas Basadas en Estándares: un camino equivocado. Educere et Educare. Revista de Educaçao,5(9),85-107. Recuperado de: https://bit.ly/2KpfjmL

Cheung, A., \& Slavin, R. E. (2013). The Effectiveness of Educational Technology Applications on Mathematics Achievement in K-12 Classrooms: A Meta-Analysis. Educational Research Review (9), 88-111. https:// doi.org/10.1016/j.edurev.2013.01.001

Cobcroft, R., Towers, S, Smith, J. \& Bruns, A. (2006). Mobile learning in review: Opportunities and challenges for learners, teachers, and institutions. In Proceedings Online Learning and Teaching (OLT) Conference 2006, pp. 21-30, Queensland University of Technology, Brisbane. Recuperado de: http:// eprints.qut.edu.au

Dale, R. (2005). Globalisation and Education: Demonstrating a «Common World Education Culture» or Locating a «Globally Structured Educational Agenda»? Educational Theory, 50, 427-448. https://doi.org/10.1111/ j.1741-5446.2000.00427.x

Darling-Hammond, L., Burns, D., Campbell,C. Goodwin, A. Hammerness, K. Low,E-L., McIntyre, A. Sato, M. \& Zeichner, K. (2017). Empowered Educators: How High-Performing Systems Shape Teaching Quality Around the World. Jossey-Bass.

Delannoy, F. y Guzmán, M. (2009). Experiencias internacionales en gestión descentralizada de la educación pública. En M. Marcel y D. Raczynski (Eds), La asignatura pendiente: claves para la revalidación de la educación pública de gestión local en Chile (pp.225-278). Uqbar. Recuperado de: https://issuu.com/ cieplan/docs/la_asignatura_pendiente

Egido, I. (2015). El liderazgo escolar en Europa. Recomendaciones y políticas para la mejora, Revista padres y maestros, 361, 18-22. https://doi.org/10.14422/pym.i361.y2015.003

Englander, E. (2017). Understanding violence. New York: Taylor\& Francis Group

Fair, H. (2019). Análisis Político del Discurso e investigación empírica: herramientas teóricas y estrategias metodológicas para estudiar identidades y procesos políticos desde América Latina. Ciencia Política, 14(27), 47-90. https://doi.org/10.15446/cp.v14n27.73323 
Fengchun M., Mark W., Hyo-Jeong S. \& Yancy T (2017). Supporting teachers with mobile technology. Lessons drawn from UNESCO projects in Mexico, Nigeria, Pakistan and Senegal. UNESCO. Recuperado de: https://bit.ly/2K5dCes

García-Aretio, L. (2017). Educación a distancia y virtual: calidad, disrupción, aprendizajes adaptativo y móvil. RIED. Revista Iberoamericana de Educación a Distancia, 20(2), 09-25. https://doi.org/10.5944/ ried.20.2.18737

García, S \& Fombona, J. (2015). Approach to the phenomenon of m-learning in English teaching. Digital Education Review, 19-36. https://doi.org/10.1344/der.2015.28.19-36

Gros, B. (2012). Retos y tendencias sobre el futuro de la investigación acerca del aprendizaje con tecnologías digitales. RED. Revista de Educación a Distancia, 32, 1-13. http://dx.doi.org/10.6018/red/50/10

Gros, B. (2015). La caída de los muros del conocimiento en la sociedad digital y las pedagogías emergentes. Education in the Knowledge Society, 16(1), 58-68. http://dx.doi.org/10.14201/eks20151615868

Gros, B. (2019). La investigación sobre el diseño participativo de entornos digitales de aprendizaje, Documents de treball / Informes (Teoria i Història de l'Educació). https://bit.ly/2VCWfo8

Gros, B., \& López, M. (2016). Students as co-creators of technology-rich learning activities in higher education. International Journal of Educational Technology in Higher Education, 13(1), 28. https://doi.org/10.1186/ s41239-016-0026-x

Hsu, Y-Ch., Ching, Y-H. \& Snelson, Ch. (2014). Research priorities in mobile learning: an international Dekphi study. CJLT, Canadian Journal of Learning and Technology, 40(2), 1-22. https://doi.org/ 10.1142/9789814619523_0001

Innerarity, D. (2019). Una teoría de la democracia compleja: Gobernar en el siglo XXI. Barcelona, Galaxia Gutenberg.

Kotthoff, H.G. (2011). Between excellence and equity: the case of the german education system. Revista Española de Educación Comparada, 18, 27-60. Recuperado de: https://bit.ly/3f0QGYp

Laclau, E. (2005). Deconstrucción, pragmatismo, hegemonía. En: C. Mouffe (Comp.). Deconstrucción y pragmatismo. Espacios del saber, 97-136.

López-García, J.N. (2018). Políticas transnacionales sobre aprendizaje móvil y educación: una selección de textos relevantes. Edutec. Revista Electrónica de Tecnología Educativa, (65), 93-109. https://doi.org/ 10.21556/edutec.2018.65.1133

Martin-Garcia, A.V. (Ed.) (2020). Blended Learning: Convergence between Technology and Pedagogy. Nueva Yprk, Springer.

Muñoz Rodríguez, J.M., Torrijos Fincias, P., Serrate González, S., Murciano Hueso, B.A. (2020). Entornos digitales, conectividad y educación: Percepción y gestión del tiempo en la construcción de la identidad digital de la juventud, Revista Española de Pedagogía, 78(277), 457-475. https://doi.org/10.22550/ REP78-3-2020-07

OCDE (2012). Equity and Quality in Education: Supporting Disadvantaged Students and Schools. OECD Publishing. Recuperado de: https://bit.ly/2KGRcN7

OCDE (2016). PISA 2015 Results (Volume II): Policies and Practices for Successful Schools, PISA. OECD Publishing. Recuperado de: https://bit.ly/2KNuRhj

OCDE (2018a). Políticas docentes efectivas: Conclusiones del informe PISA. OCDE. Recuperado de: https:// bit.ly/2SbnfZT

OCDE (2018b). TALIS - The OECD Teaching and Learning International Survey. Recueperado de: www.oecd.org/talis

OMS (2020). Alocución de apertura del Director General de la OMS en la rueda de prensa sobre la COVID-19 celebrada el 11 de marzo de 2020. Recuperado de: https://bit.ly/2zs7PK9

Pedró, F. (2017). Tecnologías para transformar la educación. Madrid, Fundación Santillana. 
Ramírez-Montoya, M. S. y García-Peñalvo, F.J. (2017). La integración efectiva del dispositivo móvil en la educación y en el aprendizaje. RIED. Revista Iberoamericana de Educación a Distancia, 20(2), 29-47. http://dx.doi.org/10.5944/ried.20.2.18884

Ramírez-Montoya, M. S., \& Ramírez- Hernández, D. C. (2016). Inverted Learning Environments with Technology, Innovation and Flexibility: Student experiences and meanings. Journal of Information Technology Research, 9(1), 18-33. https://doi.org/10.4018/jitr.2016010102

Rivera-Vargas, P., Neut, P., Luccini, P., Pascual, S., \& Prunera, P. (eds.) (2018). Pedagogías Emergentes en la Sociedad Digital. Vol. 1. Navarra, LiberLibro ediciones.

Rodríguez-Fernández, J. R. (2018). El análisis político del discurso. Apropiaciones en educación. Octaedro.

Rostan, M. \& Vaira, M., (eds.) (2011). Questioning excellence in higher education: policies, experiences and challenges in national and comparative perspective. Sense Publishers. https://doi.org/ 10.1007/978-94-6091-642-7

Sánchez-Prieto, J. C., Olmos-Migueláñez, S., y García-Peñalvo, F. J. (2017). ¿Utilizarán los futuros docentes las tecnologías móviles? Validación de una propuesta de modelo TAM extendido. RED. Revista de Educación a Distancia, 17(52). http://dx.doi.org/10.6018/red/52/5

Santiago, R. Trabaldo, S. Kamijo, M. y Fernández, A. (2015). Mobile learning: nuevas realidades en el aula. Barcelona, Océano.

Sanz-Ponce, R. y Serrano-Sarmiento, A. (2017). ¿La educación cambia? Repensando el sentido y finalidad de una escuela para todos. Teoría de la Educación. Revista Interuniversitaria, 29 (2), 167-184. http:// dx.doi.org/10.14201/teoredu292167184

SITEAL (2014). Informe 2014: Políticas TIC en los sistemas educativos de América Latina. OEI UNESCO IIPE. Recuperado de: https://bit.ly/2y8WaQ8

Torres, J.C., Infante, A. \& Torres, P.V. (2015). Aprendizaje móvil: perspectivas. RUSC. Universities and Knowledge Society Journal, 12(1), 38-49. http://dx.doi.org/10.7238/rusc.v12i1.1944

UNESCO (2012). Aprendizaje móvil para docentes. Temas globales. Recuperado de: https://bit.ly/2zxsDAa

UNESCO (2013). Directrices de la UNESCO para las políticas de aprendizaje móvil. Recuperado de: https:// bit.ly/2KC66Es

UNESCO (2017). Supporting teachers with mobile technology. Lessons drawn from unesco projects in Mexico, Nigeria, Pakistan and Senegal. Recuperado de: https://bit.ly/3eVFuMz

UNESCO (2018). UNESCO-Fazheng Project. Recuperado de: https://bit.ly/3cTiCvz

UNESCO (2020a). Apoyo de la UNESCO: respuesta del ámbito educativo de cara al COVID-19. Recuperado de: https://bit.ly/2S9cCaa

UNESCO (2020b). Soluciones para un aprendizaje a distancia. Recuperado de. https://bit.ly/3bFHpml

UNESCO (2020c). Consecuencias negativas del cierre de las escuelas. Recuperado de: https://bit.ly/3bDCOMI

Verger, A. y Normand, R. (2015). Nueva gestión pública y educación: elementos teóricos y conceptuales para el estudio de un modelo de reforma educativa global. Educaçao \& Sociedade, 132(36), 599-622. https:// doi.org/10.1590/es0101-73302015152799

Wößmann, L. 2003. Schooling Resources, Educational Institutions and Student Performance: The International Evidence. Oxford Bulletin of Economics and Statistics 65(2), 117-70. https://doi.org/ $\underline{10.1111 / 1468-0084.00045}$

Wößmann, L., Lüdemann, E., Schütz, G. \& West, M. (2007). School Accountability, Autonomy, Choice, and the Level of Student Achievement. OECD Education Working Papers. https://doi.org/10.1787/19939019 